\title{
BMJ Open How do type 2 diabetes mellitus (T2DM)-related complications and socioeconomic factors impact direct medical costs? A cross-sectional study in rural Southeast China
}

\author{
Haibin Wu, ${ }^{1}$ Karen N Eggleston, ${ }^{2}$ Jieming Zhong, ${ }^{1}$ Ruying Hu, ${ }^{1}$ Chunmei Wang, ${ }^{3}$ \\ Kaixu Xie, ${ }^{3}$ Yiwei Chen, ${ }^{4}$ Xiangyu Chen, ${ }^{1}$ Min $\mathrm{Yu}^{1}$
}

To cite: Wu H, Eggleston KN, Zhong J, et al. How do type 2 diabetes mellitus (T2DM)related complications and socioeconomic factors impact direct medical costs? A cross-sectional study in rural Southeast China. BMJ Open 2018;8:e020647. doi:10.1136/ bmjopen-2017-020647

- Prepublication history for this paper is available online. To view these files please visit the journal online (http://dx.doi org/10.1136/bmjopen-2017020647).

Received 15 November 2017 Revised 19 August 2018 Accepted 26 September 2018

Check for updates

(C) Author(s) (or their employer(s)) 2018. Re-use permitted under CC BY-NC. No commercial re-use. See rights and permissions. Published by BMJ.

For numbered affiliations see end of article.

Correspondence to

Dr Min Yu;

minyu_cdc@126.com

\section{ABSTRACT}

Objective To evaluate type 2 diabetes mellitus (T2DM)related direct medical costs by complication type and complication number, and to assess the impacts of complications as well as socioeconomic factors on direct medical costs.

Design A cross-sectional study using data from the region's diabetes management system, social security system and death registry system, 2015.

Setting Tongxiang, China.

Participants Individuals diagnosed with T2DM in the local diabetes management system, and who had 2015 insurance claims in the social security system. Patients younger than 35 years and patients whose insurance type changed in the year 2015 were excluded.

Main outcome measures The mean of direct medical costs by complication type and number, and the percentage increase of direct medical costs relative to a reference group, considering complications and socioeconomic factors.

Results A total of 19015 eligible individuals were identified. The total cost of patients with one complication was US\$1399 at mean, compared with US\$248 for patients without complications. The mean total cost for patients with 2 and $3+$ complications was US $\$ 1705$ and US\$2994, respectively. After adjustment for socioeconomic confounders, patients with one complication had, respectively, $83.55 \%$ and $38.46 \%$ greater total costs for inpatient and outpatient services than did patients without complications. The presence of multiple complications was associated with a significant $44.55 \%$ adjusted increase in total outpatient costs, when compared with one complication. Acute complications, diabetic foot, stroke, ischaemic heart disease and diabetic nephropathy were the highest cost complications. Gender, age, education level, insurance type, T2DM duration and mortality were significantly associated with increased expenditures of T2DM.

Conclusions Complications significantly aggravated expenditures on T2DM. Specific kinds of complications and the presence of multiple complications are correlated with much higher expenditures. Proper management and the prevention of related complications are urgently needed to reduce the growing economic burden of diabetes.

\section{Strengths and limitations of this study}

- This is a population-based study that estimates type 2 diabetes mellitus-related direct medical costs using insurance claims linked with healthcare management and death registry data for a large sample in rural China.

- This study is the first of its kind to examine this topic with data linking primary care management and death registry data with insurance claims for residents of rural China.

- Direct medical costs are evaluated by complication type and number, and the percentage increase in expenditures relative to the reference group, considering complications and socioeconomic factors, is assessed using generalised linear models.

- The evaluation relied on records in different electronic systems that were in some cases not aligned and whose accuracy and completeness are difficult to verify.

- The first diagnosis listed on an insurance claim was defined as the primary reason for each inpatient admission or outpatient visit, and only very limited information on self-medication costs, physical examinations and laboratory tests was found to be available.

\section{INTRODUCTION}

Diabetes is an important public health issue that adversely affects the lives of millions of individuals worldwide. ${ }^{1}$ The number of patients with diabetes has been increasing around the world, especially in China. Globally, the total number of people with diabetes is projected to rise from 415 million in 2015 to 642 million in $2040 .^{2}$ In China, a recent study reported that the overall prevalence of diabetes among adults was $10.9 \% .^{3}$

Evidence from previous studies demonstrates that diabetes imposes a substantial economic burden on individuals, their 
families, as well as healthcare systems, especially in low-income and middle-income countries. ${ }^{4}$ Moreover, diabetes-related complications obviously aggravate the economic burden of diabetes. ${ }^{5}$ In 2015 the global cost of diabetes was an estimated US $\$ 1.31$ trillion or $1.8 \%$ of global gross domestic product (GDP), and direct costs accounted for $65.3 \%$ of this total burden. ${ }^{6}$ In China, the direct medical costs of type 2 diabetes mellitus (T2DM) increased from US $\$ 2.27$ billion in 2002 to US $\$ 8.65$ billion in $2008 .^{7}$ In 2015 , the direct costs of diabetes were estimated to be US $\$ 141.58$ billion and accounted for $1.3 \%$ of GDP. ${ }^{6}$

The economic burden of diabetes has become a great challenge for clinical and public health. It is important to identify which types of complications and socioeconomic factors affect direct medical costs, so as to better understand the increasing expenditures on diabetes and to design cost-reducing diabetes management programmes. Unfortunately, few studies to date have considered the impacts of complications and socioeconomic factors on direct medical costs in rural China.

This study aims to evaluate T2DM-related direct medical costs by complication type and complication number, and to assess the impacts of complications as well as socioeconomic factors on them. To make an accurate and representative assessment, databases of primary care management, health insurance and death were linked to generate a relatively large sample of patients diagnosed with T2DM.

\section{METHODS}

\section{Data collection}

This study was conducted in Tongxiang, Zhejiang Province. This is one of the most economically prosperous areas of Southeast China, close to Shanghai and Hangzhou.

We collected data from Tongxiang's diabetes management system, social security system and death registry. The local diabetes management system and death registry are maintained by Zhejiang Provincial Centers for Disease Control and Prevention. The diabetes management system documents patients' diabetes type, date of diagnosis, demographic information (including sex, date of birth, education, etc), as well as a unique identification number. The death registry contains information on causes of death, and encompasses all deaths in the area, whether they occurred in a hospital, at home or in another community location.

The social security system is maintained by the local Social Security Bureau, and covers about $95 \%$ of registered residents. There are two basic medical insurance schemes in Tongxiang: the Urban Resident Basic Medical Insurance (URBMI) and Urban Employee Basic Medical Insurance (UEBMI). (The rural New Cooperative Medical Scheme merged into the standard URBMI in 2015.) The UEBMI includes employees and retirees from local enterprises, institutions and governmental organisations. In contrast, URBMI includes farmers, self-employed people. Compared with URBMI, UEBMI is more generous and has higher reimbursement ratios for both hospitalisation and outpatient visits. In addition to insurance type, reimbursement is determined by medication grade, hospital grade, diagnosed disease, total costs, inpatient or outpatient visits. The local Social Security Bureau has established an algorithm based on national health policies and local financial expenditures to calculate the composition of costs. People who sign up for an insurance scheme get an insurance card linked to a unique account. Generally, patients provide their insurance card to settle costs at a hospital or clinic, and an electronic system reads the information from the insurance card and calculates the total costs, including out-of-pocket (OOP) costs, instantaneously. Patients need pay only the OOP costs at that point; other costs are settled by the local Social Security Bureau. The electronic system then tracks all cost settlements. Insurance claims data include the total and OOP expenditures associated with each hospitalisation, outpatient visit and purchase of medication. Claims also specify information on insurance type, diagnosed diseases, treatment dates, the names of relevant hospitals and pharmacies.

We linked information from these three different systems using unique patient identification numbers. Individuals who met $\mathrm{WHO}$ criteria for $\mathrm{T}_{2} \mathrm{DM}^{8}$ and were registered in the local diabetes management system in 2015 were included in the study. Patients younger than 35 years old were excluded to avoid the possibility of including individuals with type 1 diabetes. Patients who did not have any insurance claim (including for diabetes, diabetes-related complications and other diseases) in 2015 or whose insurance type changed that year were also excluded.

\section{Definition of complications and mortality}

The electronic systems recorded the diagnosis and expenditure for each inpatient admission or outpatient visit. In our dataset, some diagnoses had already been encoded as ICD-10 ; the rest were documented using the Chinese name of the diagnosis. We used the first diagnosis listed on each insurance claim to define the primary reason for each visit. To standardise the diagnosis, we screened the full names and ICD-10 codes, and categorised the diagnosis into different groups. Acute diabetic complications include diabetic ketoacidosis (DKA), hyperglycaemic hyperosmolar status (HHS), lactic acidosis and hypoglycaemia coma. Chronic complications include stroke, ischaemic heart disease, retinopathy, nephropathy, neuropathy, diabetic foot and an unspecified category. Stroke includes ischaemic and haemorrhagic strokes, as well as stroke sequelae and related recovery periods, but excludes strokes caused by trauma (such as cerebral ischaemia, cerebral infarction, cerebral thrombosis, lacunar infarction and transient ischaemic attack). Ischaemic heart disease includes angina, myocardial infarction, ischaemic cardiomyopathy, patients with percutaneous 
coronary intervention (PCI) or coronary artery bypass graft, or is determined by coronary angiography, as well as sequelae, recovery period and postoperative treatment. Mortality is defined as patients with diabetes who died in 2015. If a patient with diabetes had at least one claim for a complication described above in the social security system in 2015, and the visiting date was later than the T2DM diagnosed date, then he/she was grouped in the category 'with complications'. The corresponding complication type and number were also collected for the patient. Otherwise, the patient was included in the "without complications' category.

\section{Definition of costs}

We evaluated costs using medical claims data from the social security system in 2015, based on the diagnosis noted on each visit or hospitalisation. For patients who died in 2015, we calculated the costs for the part of the year the patients were alive. The total annual costs (T2DM-related direct medical costs) were defined as the expenditures attributable to T2DM and its complications including acute diabetic complications (DKA, HHS, lactic acidosis and hypoglycaemia coma), chronic complications (stroke, ischaemic heart disease, retinopathy, nephropathy, neuropathy, diabetic foot) and an unspecified category. We excluded costs related to a diagnosis of hypertension, hyperlipidaemia, cancer, infectious diseases.

The totals costs include expenditures on diagnosis, treatment, laboratory testing, medication (prescription or non-prescription), medical materials, care provided by specialists (including diabetologists) and nurses. The OOP costs are the portion of the total costs paid by patients themselves. Inpatient and outpatient costs encompass the direct medical costs of hospital stays and outpatient visits, respectively. The self-medication cost is defined as non-prescription drug purchases made by the patient, for example, at pharmacies outside of hospitals or clinics.

\section{Statistical analysis}

Statistical analyses were conducted using SAS analytical software (V.9.2, SAS Institute). All statistical significance decisions were based on two-tailed $\mathrm{p}$ values below 0.05 . Descriptive statistics for demographic information use frequency, percentages, mean and SD. We compared binomial and multinomial variables with Pearson's $\chi^{2}$ test, and ordinal variables with the Cochran-Armitage test. Because the resulting distribution of costs was skewed, we calculated the mean, median and quartile of unit total and OOP costs by complication type and number. We also presented 95\% CIs of means that are bootstrapped with 1000 bootstrap samples. Individuals who did not have an insurance claim due to T2DM and its related complications were treated as US\$0.0015 (ie, ¥0.01, US $\$ 1 \sim ¥ 6.5$ based on the exchange rate in 2015) in the calculation.

In order to estimate the impacts of complications on inpatient and outpatient costs while controlling for demographic information and to account for the skewed distribution of costs, we used generalised linear models (GLMs) with gamma distribution and a log-link function to estimate multivariable regressions. The dependent variables were the total costs and OOP costs for both inpatient and outpatient care. The presented estimates were exponentiated. As a result, estimated coefficients represent percentage differences relative to the reference group. Moreover, GLMs were also used to compare cost patterns across different categories with adjustments of age, sex, insurance type, education, income, occupation, T2DM duration and mortality.

\section{Patient and public involvement}

We linked the information from three different electronic systems and did not seek direct information from individuals. In other words, there was no patient or public involvement in the study.

\section{RESULTS}

Among the 21308 managed patients with diabetes, 1721 cases did not have any insurance claim (including for diabetes, diabetes-related complications and other diseases) in 2015. In addition, 253 patients were diagnosed with type 1 diabetes, 235 patients were younger than 35 years and 84 cases involved an insurance change in 2015. After excluding these cases, there were 19015 individuals eligible for analysis (see figure 1). Among them, 16675 individuals $(87.7 \%$ ) had an insurance claim due to T2DM or related complications. Of these, 16275 $(97.6 \%)$ patients had outpatient claims, compared with $1419(8.5 \%)$ who had inpatient claims. Table 1 shows that $74.93 \%$ of patients had URBMI, and these had a significantly lower age than those with UEBMI (mean \pm SD age: $62.73 \pm 11.11$ years vs $64.17 \pm 11.42$ years, $\mathrm{p}=0.02$, respectively). Compared with female patients, male patients had a significantly greater proportion of UEBMI $(p<0.001)$. Additionally, on average, patients under UEBMI had attained a higher level of education than those under URBMI $(p<0.001)$. There was no significant difference in mortality between patients with UEBMI and URBMI. The registered prevalence of complications in the study population was $12.25 \%$ (95\% CI $11.78 \%$ to $12.71 \%$ ). Compared with patients under URBMI, patients under UEBMI had significantly higher rates of ischaemic heart disease, stroke, nephropathy and the 'unspecified' category of complications. In our sample, $5.89 \%$ of cases lacked information on education level and $10.23 \%$ on income.

Among patients with complications, 87.63\% (2041 patients) had complications in one category, and the costs due to diabetes and its complications totalled US\$2.86 million. Of this, $50.19 \%$ was for patients with stroke, $36.19 \%$ for patients with ischaemic heart disease, while other complications accounted for less than $5 \%$ each. Table 2 shows the annual total and OOP costs per patient for different types of complications. The unit 


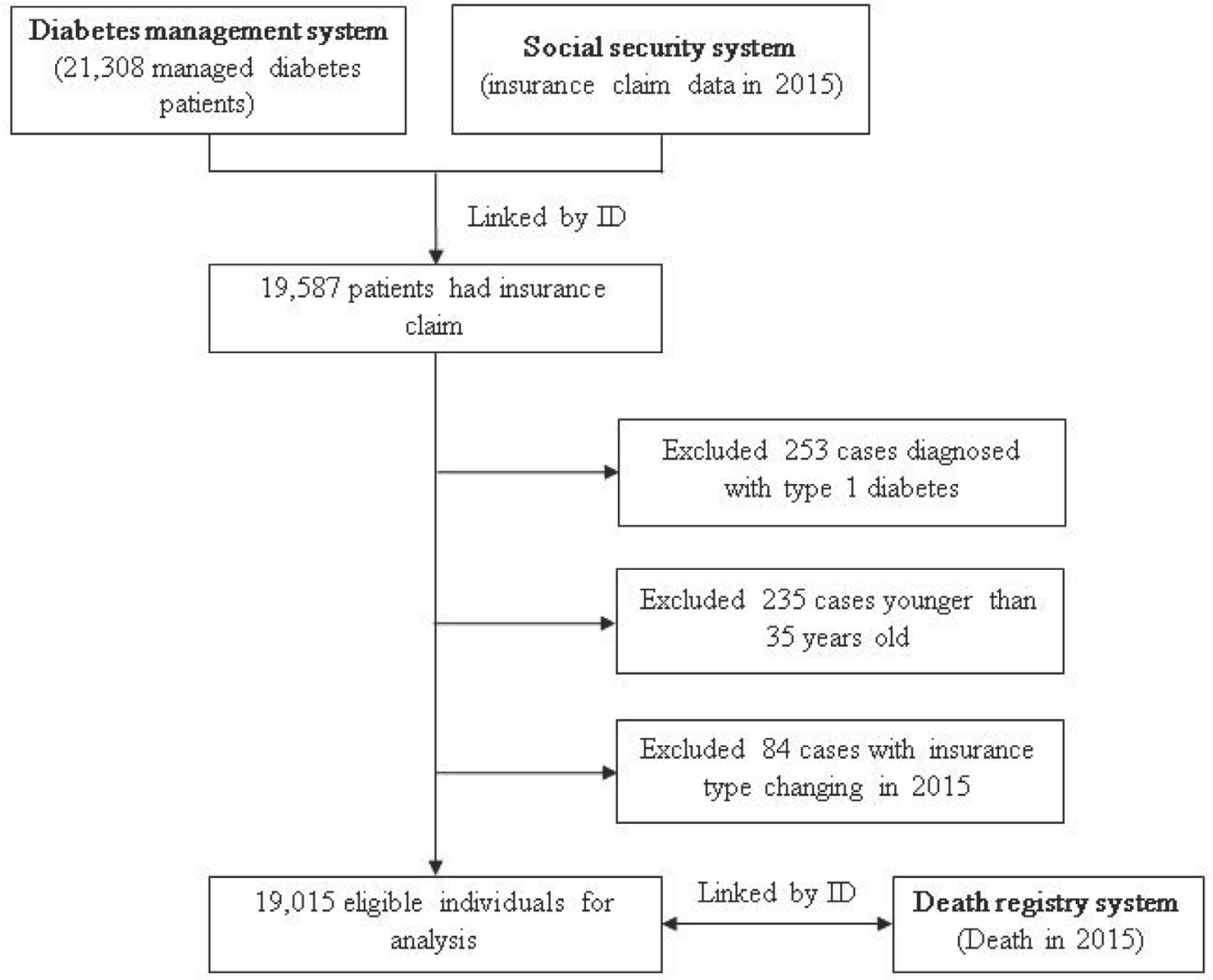

Figure 1 The diagram of the final dataset selection.

total expenditures of patients with one complication were US $\$ 1399$ at mean (95\% CI US $\$ 1222$ to US $\$ 1591$ ), compared with $\$ 248$ (95\% CI US\$242 to US\$255) for patients without complications. The mean OOP expenditures were US\$525 (95\% CI US\$472 to US\$584) and US $\$ 105$ (95\% CI US $\$ 102$ to US $\$ 108)$ for patients with one or no complication, respectively. There were statistically significant differences in both total and OOP costs among patients with different complications (GLM regression, total cost: $\chi^{2}=56.25, \mathrm{p}<0.001 ;$ OOP cost: $\left.\chi^{2}=22.53, p=0.02\right)$. Patients who suffered acute complications had the highest mean expenditures of US $\$ 5558$ (95\% CI US $\$ 1806$ to US $\$ 12393$ ), followed by those with diabetic foot (US $\$ 5207,95 \%$ CI US $\$ 2067$ to US $\$ 9647$ ), stroke (US\$1724, 95\% CI US $\$ 1420$ to US $\$ 2145$ ), nephropathy (US $\$ 1692,95 \%$ CI US $\$ 1253$ to US $\$ 2255$ ), ischaemic heart disease (US\$1150, 95\% CI US $\$ 940$ to US $\$ 1413$ ), neuropathy (US\$1102, 95\% CI US\$691 to US\$1628), unspecified (US\$977, 95\% CI US $\$ 706$ to US\$1272) and retinopathy (US\$766, 95\% CI US $\$ 617$ to US $\$ 933$ ) (see table 2).

Among patients with one category of complication, the expenditures associated with that complication accounted for $70.98 \%$ of their total medical expenditure. As figure 2 shows, patients with acute complications spent $81.71 \%$ of their total costs on the treatment of these complications. The proportion of total expenditures associated with T2DM-related chronic complications was as follows: stroke $78.81 \%$, diabetic foot $74.79 \%$, ischaemic heart disease $72.53 \%$, nephropathy $33.12 \%$, unspecified $29.33 \%$, neuropathy $27.53 \%$ and retinopathy $19.58 \%$.

Figure 3 shows that the mean of total cost and OOP cost per patient increased with the number of complications. The mean total cost for patients with two complications was US\$1705 (95\% CI US\$1405 to US\$2048), compared with US $\$ 2994$ (95\% CI US $\$ 1847$ to US $\$ 4232$ ) for patients with 3+ complications. Moreover, patients with 2 and $3+$ complications had a mean OOP cost of US\$666 (95\% CI US $\$ 544$ to US $\$ 812$ ) and US $\$ 1055$ (95\% CI US $\$ 616$ to US\$1441), respectively. The expenditures of patients with 1,2 and $3+$ complications differed at statistically significant levels for both total costs (GLM regression, $\chi^{2}=19.48$, $\mathrm{p}<0.001$ ) and OOP costs (GLM regression, $\chi^{2}=23.30$, $\mathrm{p}<0.001)$.

Controlling for age, sex, educational level, income, occupation, insurance type, duration of T2DM diagnosis and mortality, patients with one category of complication had $83.55 \%(\mathrm{p}<0.001)$ and $38.46 \% \quad(\mathrm{p}<0.001)$ higher total inpatient and outpatient expenditures, respectively, compared with patients without complications. Estimates also show that patients with diabetic foot, acute complications, stroke, ischaemic heart disease, nephropathy, neuropathy, retinopathy and unspecified complications had inpatient expenditures that were, respectively, $140.80 \%, 116.75 \%, 86.30 \%, 86.12 \%$, 52.93\%, $51.86 \%$, $51.70 \%$ and $48.44 \%$ higher than those of similar patients without complications $(\mathrm{p}<0.05)$. In addition, outpatient expenditures were $86.86 \% \quad(\mathrm{p}<0.05)$ higher among 
Table 1 Characteristics of study population by insurance type

\begin{tabular}{llccc} 
Characteristics & Overall & UEBMI & URBMI & $14248(74.93)$ \\
\hline N & $19015(100.00)$ & $4767(25.07)$ & P values & $<0.001^{*}$
\end{tabular}

\begin{tabular}{|c|c|c|c|c|}
\hline $35-50$ & 11.68 & 10.17 & 12.18 & \\
\hline $50-59$ & 26.72 & 24.96 & 27.30 & \\
\hline $60-69$ & 33.13 & 32.22 & 33.43 & \\
\hline Mean (SD) & $63.09(11.21)$ & $64.17(11.42)$ & $62.73(11.11)$ & $0.020 \dagger$ \\
\hline Gender, \% & & & & $<0.001 \neq$ \\
\hline Female & 56.01 & 37.55 & 62.19 & \\
\hline Education level, \% & & & & $<0.001 \neq$ \\
\hline No formal school & 34.42 & 10.59 & 42.39 & \\
\hline Primary school & 38.35 & 36.63 & 38.92 & \\
\hline Middle school or higher & 21.34 & 47.51 & 12.58 & \\
\hline $231-461$ & 55.43 & 52.34 & 56.46 & \\
\hline$\geq 462$ & 30.28 & 33.54 & 29.19 & \\
\hline Unspecified & 10.23 & 11.81 & 9.71 & \\
\hline Occupation, \% & & & & $<0.001 \neq$ \\
\hline Worker & 15.98 & 26.77 & 12.37 & \\
\hline Farmer & 52.04 & 15.46 & 64.28 & \\
\hline Retired/housework & 15.55 & 36.33 & 8.60 & \\
\hline Others & 16.43 & 21.44 & 14.75 & \\
\hline Acute complications & 0.07 & 0.04 & 0.08 & $0.352 \ddagger$ \\
\hline Ischaemic heart disease & 5.98 & 8.12 & 5.26 & $<0.001 \ddagger$ \\
\hline Stroke & 5.55 & 6.50 & 5.23 & $0.001 \ddagger$ \\
\hline Retinopathy & 0.88 & 1.05 & 0.83 & $0.159 \ddagger$ \\
\hline Neuropathy & 0.27 & 0.25 & 0.27 & $0.799 \ddagger$ \\
\hline Nephropathy & 0.42 & 0.80 & 0.29 & $<0.001 \ddagger$ \\
\hline Diabetic foot & 0.03 & 0.02 & 0.03 & $0.794 \ddagger$ \\
\hline Unspecified & 0.64 & 1.24 & 0.44 & $<0.001 \ddagger$ \\
\hline
\end{tabular}

${ }^{*}$ Cochran-Armitage test.

†Student's t-test.

$\ddagger$ Pearson's $\chi^{2}$ test.

T2DM, type 2 diabetes mellitus; UEBMI, Urban Employee Basic Medical Insurance; URBMI, Urban Resident Basic Medical Insurance.

patients with acute complications. For chronic complications, the outpatient expenditures associated with diabetic nephropathy, retinopathy, the unspecified category, neuropathy, stroke and ischaemic heart disease were higher by $96.86 \%, 77.80 \%, 63.45 \%, 47.60 \%, 33.31 \%$ and $26.63 \%$, respectively $(\mathrm{p}<0.05)$. Despite these differences, 
Table 2 The annual direct medical costs per patient by complication type (US\$)

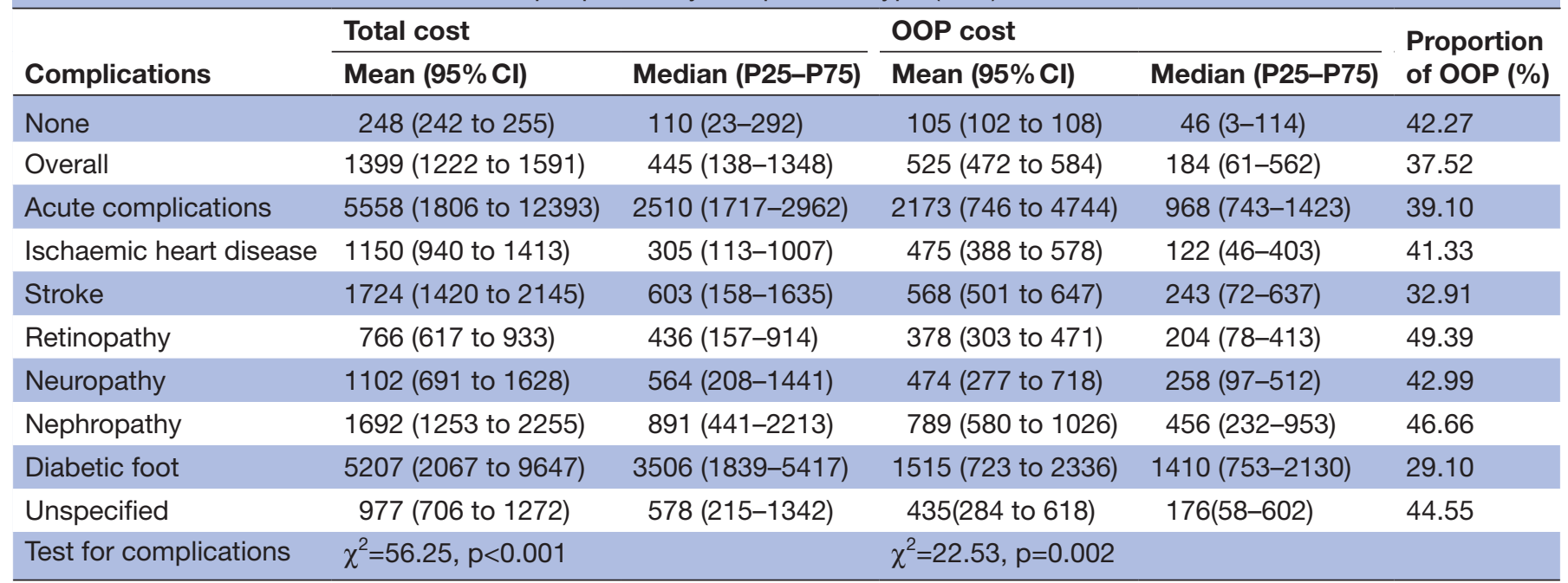

Only patients without any complication or those with one category of complication have been included in this table. A total of 2340 patients who did not have T2DM-related insurance claims were treated as US $\$ 0.0015$ in calculation. P value was estimated by GLM regression with adjustments for age, sex, insurance type, education, income, occupation, T2DM duration and mortality.

GLM, generalised linear model; OOP, out-of-pocket; T2DM, type 2 diabetes mellitus.

no statistically significant increase in outpatient costs was observed among patients with diabetic foot. Unsurprisingly, complications also significantly increased the OOP costs associated with both inpatient admissions and outpatient visits, except those for diabetic foot (given the small number of cases) (see table 3 ).
Compared with patients under UEBMI, those under URBMI had higher outpatient costs and lower inpatient costs, but higher OOP costs for both inpatient and outpatient visits. Moreover, male patients, patients with high education levels and patients with a long duration of T2DM had significantly higher total and OOP costs

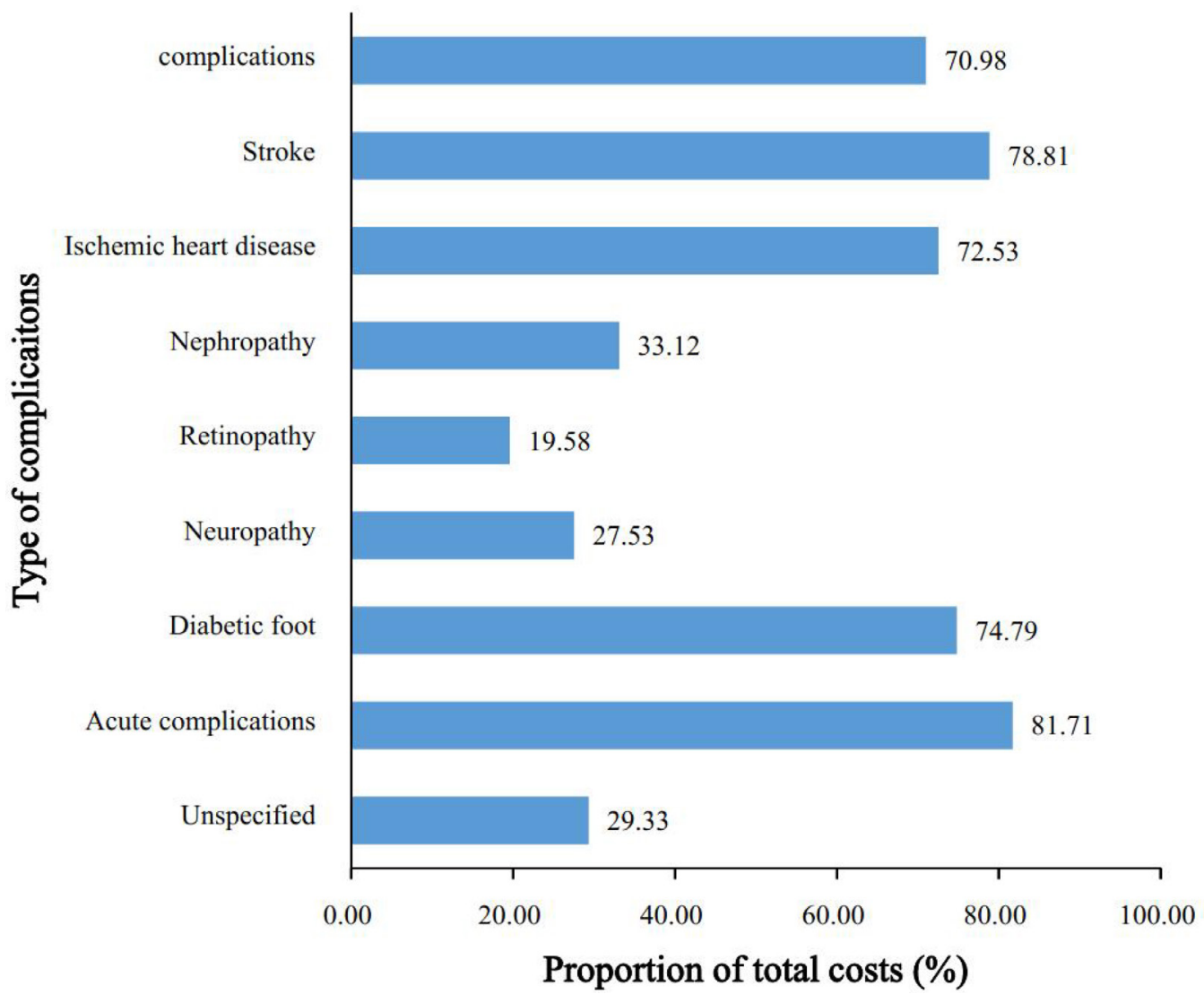

Figure 2 Proportion (\%) of total costs attributable to complication treatment (only patients with complication in one category have been included in this figure). 


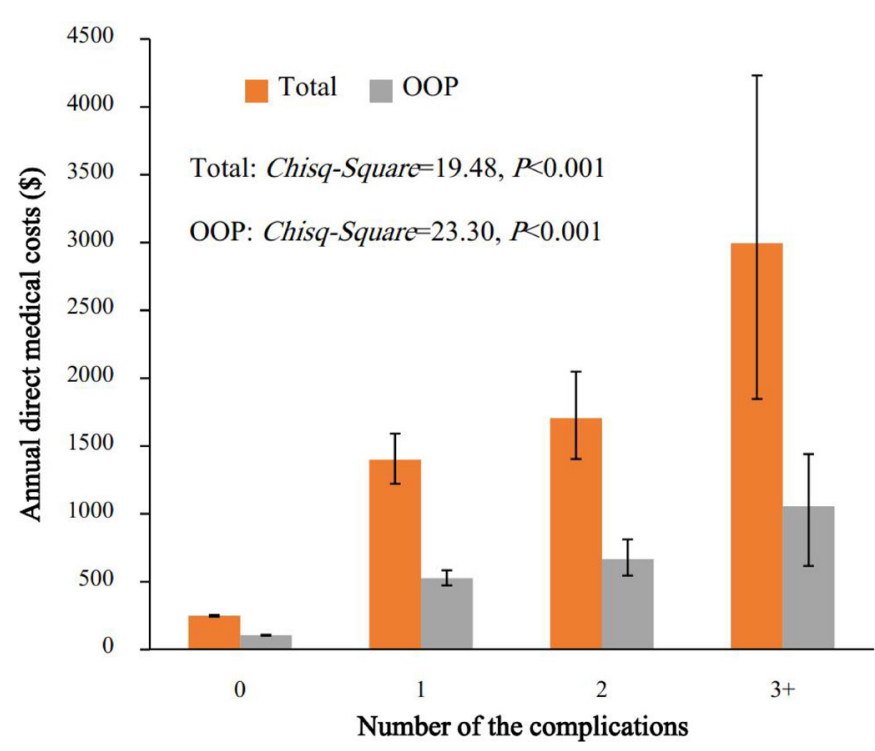

Figure 3 Mean of total costs and OOP costs with bootstrapped $95 \% \mathrm{Cl}$ among patients with diabetes with different numbers of complications. $P$ value was estimated by GLM regression with adjustments for age, sex, insurance type, education, income, occupation, T2DM duration and mortality. GLM, generalised linear model; OOP, out-ofpocket; T2DM, type 2 diabetes mellitus.

for both inpatient and outpatient visits than their counterparts. In addition, we found that inpatient total costs were positively associated with age, and patients who were farmers had the lowest outpatient costs. In addition, patients who died in 2015 had higher inpatient costs but lower outpatient costs (see table 3 ).

Table 4 shows that the total and OOP inpatient expenditures of those with more than one complication were higher than those of patients with a single complication, but the difference was not statistically significant. Despite this, having more than one complication was associated with $44.55 \%(\mathrm{p}<0.001)$ higher outpatient total costs and $44.36 \%(\mathrm{p}<0.001)$ higher outpatient OOP costs.

\section{DISCUSSION}

This is the first population-based study to provide estimates using insurance claims linked with death registry and healthcare management data for a large sample in rural China. The findings provide valuable evidence for policy-makers and managers on the high expenditures and opportunity costs associated with managing patients with diabetes and its complications.

\section{Impacts of complication type on direct medical costs}

We found that complications were a significant determinant of variations in both inpatient and outpatient costs. The mean cost for patients with one complication was about US\$1399 in our study sample, which is far less than in high-income Western countries, such as Denmark, where healthcare costs for similar patients range from about US $\$ 2780$ to US $\$ 7255$ person-years. ${ }^{9}$ However, it is comparable to or higher than the expenditure observed in other middle-income countries, such as Thailand, where the median cost for an individual with T2DM with complications is US $\$ 480 .{ }^{10}$ After adjustment for other potential confounders, patients with one category of complications had, respectively, $83.55 \%$ and $38.46 \%$ greater total costs for inpatient and outpatient services than did patients without complications. This finding is in concordance with a study conducted in Spain, which found 1.6 times greater resource use for patients with microvascular complications and 2.3 times for those with macrovascular complications. ${ }^{11}$ An earlier study in China also reported that in the presence of microvascular and macrovascular complications, the median direct medical cost was 1.5 times and 1.7 times higher, respectively, than for patients with no complications. ${ }^{12}$ Other studies find similar results. ${ }^{13-15}$

It is important to identify which types of complications greatly increase the economic burden, so as to incorporate them into cost-reducing diabetes management programmes. In our study, patients with acute complications had the highest mean cost of US $\$ 5558$, which was higher than the cost of DKA in some African countries (ranging from US\$39 in Guinea to US\$1100 in Benin). ${ }^{16}$ In addition, we found that patients with diabetic foot spent about US\$5207 at mean. Cavanagh et al reported that the cost of treatment ranged from US\$3959 to US $\$ 188645$ for diabetic foot ulcers in theUSA, and it ranged from US\$102 to US\$3060 in Tanzania. ${ }^{17}$ For cardiovascular complications, the mean cost was US $\$ 1150$ and US $\$ 1724$ for ischaemic heart disease and stroke, respectively. Similarly, Caporale et al estimated the cost of hospitalisation for cardiovascular complications in Argentina to be US $\$ 1628 .^{18}$ Nevertheless, a study in United Arab Emirates estimated that the average annual treatment costs of heart attack and stroke were US $\$ 5557$ and US $\$ 7261$, respectively. ${ }^{19}$ Not surprisingly, the impacts on direct medical costs vary widely by type of complication. Controlling for other explanatory variables, patients with diabetic foot, acute complications, stroke and ischaemic heart disease were associated with particularly high inpatient costs (increasing by $140.80 \%, 116.75 \%, 86.30 \%$ and $86.12 \%$, respectively), compared with patients without complications. Among outpatients with diabetes, diabetic nephropathy, acute complications and retinopathy involved the greatest economic burden (increasing by $96.86 \%, 86.86 \%$ and $77.80 \%$, respectively, over those without complications). The cost due to complications, as a proportion of total costs, also increased. This might indicate that the severity of complications increased over time, and is consistent with previous studies in Western countries, which indicate that renal and cardiovascular complications are the most prevalent and are associated with particularly high costs. ${ }^{20}{ }^{21} \mathrm{~A}$ hospital-based study in Hangzhou reported that patients with coronary artery disease and diabetic foot had, respectively, 93.7\% and $89.0 \%$ higher inpatient costs. ${ }^{22}$ A study in English hospitals also found that patients who suffered diabetic foot and lower limb had significantly higher costs than 
Table 3 The impacts of complication type and socioeconomic factors on direct medical costs

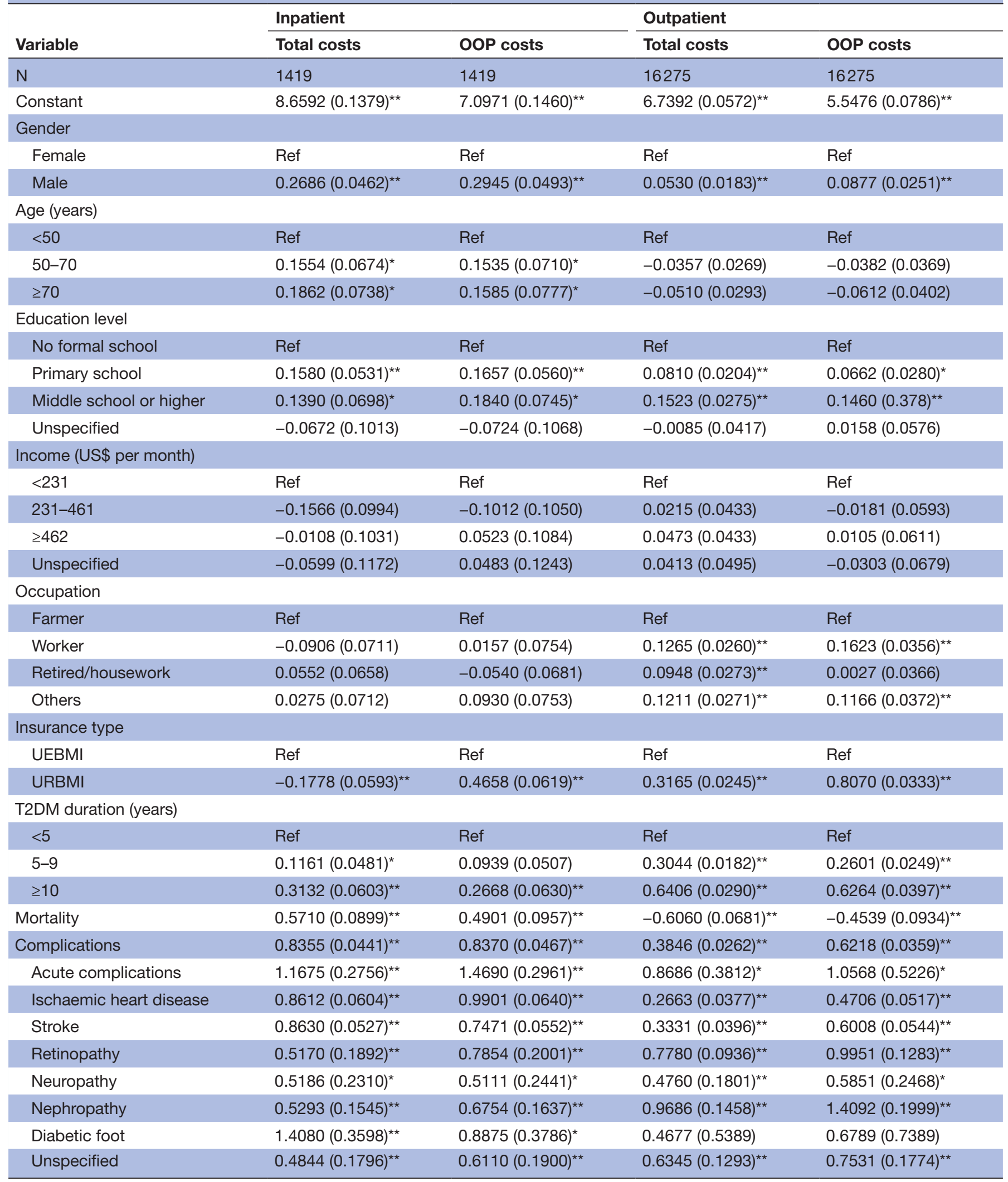

Data, patients with one category complication and those without any complication in 2015. Econometric model: GLM regression with total costs and OOP costs as the dependent variable for inpatient admissions and outpatient visits, respectively. A total of 1219 patients who spent US $\$ 0$ for OOP costs were treated as US\$0.0015 in GLM regression. The numbers in the table are GLM regression coefficients. SEs in parentheses. ${ }^{*} \mathrm{P}<0.05 ;{ }^{*} \mathrm{P}<0.01$.

GLM, generalised linear model; OOP, out-of-pocket; T2DM, type 2 diabetes mellitus; UEBMI, Urban Employee Basic Medical Insurance; URBMI, Urban Resident Basic Medical Insurance. 
Table 4 The adjusted impacts of complication number on direct medical costs

\begin{tabular}{|c|c|c|c|c|}
\hline \multirow[b]{2}{*}{ Variable } & \multicolumn{2}{|l|}{ Inpatient } & \multicolumn{2}{|l|}{ Outpatient } \\
\hline & Total costs & OOP costs & Total costs & OOP costs \\
\hline $\mathrm{N}$ & 839 & 839 & 2262 & 2262 \\
\hline \multicolumn{5}{|l|}{ Complication no } \\
\hline One complication & Ref & Ref & Ref & Ref \\
\hline
\end{tabular}

Data, patients with one and multiple category complications in 2015. Econometric model: GLM regression with total costs and OOP costs as the dependent variable for inpatient admissions and outpatient visits, respectively. The model adjusted for sex, age, education level, income, occupation, insurance type, T2DM duration and mortality. The numbers in the table are GLM regression coefficients. SEs in parentheses. ${ }^{* *} \mathrm{P}<0.01$.

GLM, generalised linear model; OOP, out-of-pocket; T2DM, type 2 diabetes mellitus.

other patients. ${ }^{23}$ Similarly, a study in the USA indicated that coronary heart disease, congestive heart failure and amputation were each associated with $70 \%-150 \%$ higher medical costs. ${ }^{24}$ Acute complications and an acute attack of ischaemic heart disease and stroke are commonly severe, and require surgery, PCI or other special treatments in a hospital. Diabetic foot and nephropathy seriously affect the quality of patients' lives, and patients have to go to hospitals frequently to obtain medical services. Note that the outpatient expenditures associated with stroke and ischaemic heart disease were higher by $33.31 \%$ and $26.63 \%$, respectively $(\mathrm{p}<0.05)$; these increases are lower than for other complications. The outpatient visits for ischaemic heart disease and stroke covered treatment during the postoperative and recovery period. Moreover, after being admitted to a hospital for an acute episode, many rural patients, who usually must pay a higher share of treatment costs relative to their income, choose to be rehabilitated at home, thus reducing the sample mean outpatient cost.

\section{Impacts of number of complications on direct medical costs}

Many previous studies have demonstrated that the number of complications has a significant impact on diabetic expenditure. ${ }^{195-27}$ In our study, the mean total cost for patients with 2 and $3+$ complications was US $\$ 1705$ and US\$2994, respectively. Morsanutto et al estimated the annual medical costs for patients in Italy with 1 and $2+$ complications at US $\$ 2296$ and US $\$ 3989$, respectively. ${ }^{28}$ In particular, the presence of more than one complication was associated with a significant $44.55 \%$ adjusted increase in outpatient total costs, when compared with one complication. According to a study in four major cities in China, for each increase in the number of complications, there was an approximately $33 \%$ increase in annual direct medical costs. ${ }^{29}$ Similarly, in Brazil, outpatient care costs for patients with two types of complications were $25 \%$ higher than for those with one. ${ }^{30}$

Impacts of socioeconomic factors on direct medical costs Compared with patients under UEBMI, those under URBMI had higher outpatient costs and lower inpatient costs, but higher OOP costs for both inpatient and outpatient visits. This pattern reflects the relative generosity of UEBMI relative to the insurance for rural residents (the majority of those covered by Tongxiang's URBMI), and is consistent with several previous studies including Wang et al documenting differences the annual direct medical costs of T2DM among patients enrolled in different insurance schemes. ${ }^{29}$ In Tongxiang, UEBMI covers more services with a higher reimbursed proportion than URBMI, and hospital admissions generally have better insurance coverage (ie, a higher reimbursement ratio) than outpatient visits do. Patients under UEBMI often live in urban areas, benefit from better sanitation services and have easy access to higher level medical providers such as urban hospitals. The results of our study imply that patients with UEBMI are prone to have more hospitalisations than similar patients with different insurance, which might indicate some inefficient overuse (or 'moral hazard').

It was reported in a study of the USA that men had more hospitalisations, longer stays and higher costs than women. ${ }^{31}$ On the contrary, some previous studies in China reported that women's diabetes disease burden was heavier than that of men. ${ }^{31} 32$ In our study, male patients are more like to have higher expenditures for both inpatient and outpatient visits than their female counterparts. These inconsistencies may be because of differences among healthcare systems, cost definitions, methodologies and demographic characteristics. In rural China, males are often the main source of family income; they represent a significantly greater proportion of UEBMI and have higher education levels than do females. Therefore, males might pay much more attention to their healthcare and have a higher willingness to pay. In addition to gender, the duration of T2DM is seen to matter. As patients continue to suffer T2DM over time, the costs for both inpatient and outpatient visits rise significantly. T2DM is a chronic medical condition, and the risk of developing complications increases with time. ${ }^{33}$ Also, we found total inpatient costs to be positively associated with age, which is in accordance with previous research. ${ }^{22} 32$ 
Studies have shown that age is an important risk factor for many chronic diseases, including diabetes. ${ }^{34} 35$ Besides, elderly patients with diabetes are more likely to have diabetes-related complications. ${ }^{36-38}$ Consequently, age substantially increases the burden of diabetes that is driving expenditure. Compared with patients with no formal education, patients with a higher education level might have higher income and are more likely to be under UEBMI. Both their economic status and UEBMI's higher reimbursement level might encourage them to use more health services and spend more money on healthcare. Similarly, in the case of occupation, it is likely that expenditure is driven partially by the higher reimbursement level of UEBMI and the higher income of formal employees compared with farmers.

As has been noted, patients who died in 2015 had higher inpatient costs but lower outpatient costs. We inferred that patients generally had severe complications or comorbidity before death and hospitalisation was needed, but after that, outpatient costs did not increase. This implies that healthcare practitioners should pay more attention to patients with severe complications or comorbidity and reinforce the management of these patients to better control their glucose levels.

\section{Limitations}

There were several limitations to this study. First, it relies on the accuracy and completeness of data drawn from several electronic systems. Second, the first diagnosis listed on an insurance claim was used as the primary reason for each inpatient admission or outpatient visit. Thus, the study only imperfectly distinguishes the allocation of expenditures between complication-related and non-complication-related causes. Third, the study defines patients with complications as those who had at least one claim for a complication in the social security system in 2015. This could have led to an underestimation of the prevalence of complications. Therefore, for some kinds of complications, especial rare ones, the estimates in the models have relatively large SEs. Fourth, the study lacked data on physical examinations and laboratory tests, which might lead to underestimation of diabetes expenditures. Finally, the URBMI in Tongxiang did not collect information on self-medication, and the self-medication records in UEBMI were limited. Hence, these expenditures, which are a part of direct medical costs, could not be included in the study.

\section{CONCLUSIONS}

The results indicated that T2DM-related complications significantly aggravated the economic burden of diabetes. Specific kinds of complications and the presence of multiple complications are correlated with much higher expenditures. Acute complications, diabetic foot, stroke, ischaemic heart disease and diabetic nephropathy were the highest cost complications. Gender, age, education level, insurance type, T2DM duration and mortality were significant predictors of differences in T2DM costs. As diabetes continues to become more prevalent in China, its proper management and the prevention of related complications are urgently needed to reduce the growing economic burden of the disease.

\section{Author affiliations}

${ }^{1}$ Department of NCDs Control and Prevention, Zhejiang Provincial Center for Disease Control and Prevention, Hangzhou, China

${ }^{2}$ Shorenstein Asia-Pacific Research Center, Stanford University, Stanford, California, USA

${ }^{3}$ Tongxiang Center for Disease Control and Prevention, Jiaxing, China

${ }^{4}$ Department of Economics, Stanford University, Stanford, California, USA

Acknowledgements We would like to thank the Social Security Bureau in Tongxiang. We also thank Pedro Gallardo from Stanford University.

Contributors Research area and study design: MY, KNE and HW; data acquisition and management: $\mathrm{CW}, \mathrm{KX}$ and $\mathrm{XC}$; statistical analysis: HW; interpretation and writing of the article: HW, KNE and MY. JZ, RH and YC were involved in the critical revision of the article, and its interpretation and design. Each author contributed important intellectual content during manuscript drafting or revision and all authors have seen and approved the final version.

Funding This study was supported by the Chinese Ministry of Science and Technology's National Key Research and Development Program, 'precision medical research' (2016YFC0901200, 2016YFC0901205); the National Natural Science Foundation of China (81803314); Medicine and Health Care in Zhejiang Province, Science and Technology Platform Project (2018RC025, 2017RC017); the Public Technology Applications Research Program of Zhejiang Province (2017C33090).

Competing interests None declared.

Patient consent Not required.

Ethics approval This study was approved by the Ethics Committee of Zhejiang Provincial Centers for Disease Control and Prevention

Provenance and peer review Not commissioned; externally peer reviewed. Data sharing statement No additional data are available.

Open access This is an open access article distributed in accordance with the Creative Commons Attribution Non Commercial (CC BY-NC 4.0) license, which permits others to distribute, remix, adapt, build upon this work non-commercially, and license their derivative works on different terms, provided the original work is properly cited, appropriate credit is given, any changes made indicated, and the use is non-commercial. See: http://creativecommons.org/licenses/by-nc/4.0/.

\section{REFERENCES}

1. Zimmet PZ, Magliano DJ, Herman WH, et al. Diabetes: a 21st century challenge. Lancet Diabetes Endocrinol 2014;2:56-64.

2. International Diabetes Federation (IDF). IDF diabetes atlas. 7th edn, 2015. (Accessed 17 Oct 2017).

3. Wang L, Gao P, Zhang M, et al. Prevalence and ethnic pattern of diabetes and prediabetes in China in 2013. JAMA 2017;317:2515-23.

4. Wild S, Roglic G, Green A, et al. Global prevalence of diabetes: estimates for the year 2000 and projections for 2030. Diabetes Care 2004;27:1047-53.

5. Ettaro L, Songer TJ, Zhang P, et al. Cost-of-illness studies in diabetes mellitus. Pharmacoeconomics 2004;22:149-64.

6. Bommer C, Heesemann E, Sagalova V, et al. The global economic burden of diabetes in adults aged 20-79 years: a cost-of-illness study. Lancet Diabetes Endocrinol 2017;5:423-30.

7. Hu H, Sawhney M, Shi L, et al. A systematic review of the direct economic burden of type 2 diabetes in China. Diabetes Ther 2015;6:7-16.

8. World Health Organization (WHO). Definition, diagnosis and classification of diabetes mellitus and its complications: report of a WHO Consultation. Part 1. Diagnosis and classification of diabetes Mellitus. Geneva: World Health Organization, 1999.

9. Sortsø C, Green A, Jensen PB, et al. Societal costs of diabetes mellitus in Denmark. Diabet Med 2016;33:877-85.

10. Deerochanawong C, Ferrario A. Diabetes management in Thailand: a literature review of the burden, costs, and outcomes. Global Health 2013;9:11. 
11. Mata $\mathrm{M}$, Antoñanzas $F$, Tafalla $\mathrm{M}$, et al. [The cost of type 2 diabetes in Spain: the CODE-2 study]. Gac Sanit 2002;16:511-20.

12. Wang W, Fu CW, Pan CY, et al. How do type 2 diabetes mellitusrelated chronic complications impact direct medical cost in four major cities of urban China? Value Health 2009;12:923-9.

13. Brown JB, Nichols GA, Glauber HS, et al. Health care costs associated with escalation of drug treatment in type 2 diabetes mellitus. Am J Health Syst Pharm 2001;58:151-7.

14. Henriksson F, Agardh CD, Berne C, et al. Direct medical costs for patients with type 2 diabetes in Sweden. $J$ Intern Med 2000;248:387-96.

15. Leśniowska J, Schubert A, Wojna $M$, et al. Costs of diabetes and its complications in Poland. Eur J Health Econ 2014;15:653-60.

16. Mutyambizi C, Pavlova M, Chola L, et al. Cost of diabetes mellitus in Africa: a systematic review of existing literature. Global Health 2018;14:3.

17. Cavanagh $P$, Attinger $C$, Abbas $Z$, et al. Cost of treating diabetic foot ulcers in five different countries. Diabetes Metab Res Rev 2012;28(Suppl 1):107-11.

18. Caporale JE, Elgart JF, Gagliardino JJ. Diabetes in Argentina: cost and management of diabetes and its complications and challenges for health policy. Global Health 2013;9:54.

19. Al-Maskari F, El-Sadig M, Nagelkerke N. Assessment of the direct medical costs of diabetes mellitus and its complications in the United Arab Emirates. BMC Public Health 2010;10:679.

20. Brown JB, Pedula KL, Bakst AW. The progressive cost of complications in type 2 diabetes mellitus. Arch Intern Med 1999;159:1873-80.

21. Smith TL, Melfi CA, Kesterson JA, et al. Direct medical charges associated with myocardial infarction in patients with and without diabetes. Med Care 1999;37:AS4-AS11.

22. Li H, Chen BK, Shah N, et al. Socioeconomic correlates of inpatient spending for patients with type 2 diabetes mellitus in China: evidence from Hangzhou. Exp Clin Endocrinol Diabetes 2012;120:35-44.

23. Kristensen T, Laudicella M, Ejersted C, et al. Cost variation in diabetes care delivered in English hospitals. Diabet Med 2010;27:949-57.

24. Li R, Bilik D, Brown MB, et al. Medical costs associated with type 2 diabetes complications and comorbidities. Am J Manag Care 2013;19:421-30

25. Massi-Benedetti M. CODE-2 Advisory Board, The cost of diabetes type II in Europe: the CODE-2 study. Diabetologia 2002;45:S1-4.
26. Morsanutto A, Berto P, Lopatriello S, et al. Major complications have an impact on total annual medical cost of diabetes: results of a database analysis. J Diabetes Complications 2006;20:163-9.

27. Kim TH, Chun KH, Kim HJ, et al. Direct medical costs for patients with type 2 diabetes and related complications: a prospective cohort study based on the Korean National Diabetes Program. J Korean Med Sci 2012;27:876-82.

28. Morsanutto A, Berto P, Lopatriello S, et al. Major diabetes complications have an impact on total annual medical cost of type 2 diabetes. Diabetes Care 2004;27:1841-2.

29. Wang W, Fu C, Zhuo H, et al. Factors affecting costs and utilization of type 2 diabetes healthcare: a cross-sectional survey among 15 hospitals in urban China. BMC Health Serv Res 2010;10:244.

30. Bahia LR, Araujo DV, Schaan BD, et al. The costs of type 2 diabetes mellitus outpatient care in the Brazilian public health system. Value Health 2011;14:S137-S140.

31. Cook CB, Naylor DB, Hentz JG, et al. Disparities in diabetes-related hospitalizations: relationship of age, sex, and race/ethnicity with hospital discharges, lengths of stay, and direct inpatient charges. Ethn Dis 2006;16:126-31.

32. Wang $\mathrm{H}$, Lin $\mathrm{X}$, Zhang $\mathrm{Z}$, et al. The economic burden of inpatients with type 2 diabetes: a case study in a Chinese hospital. Asia Pac $J$ Public Health 2015;27:49S-54.

33. Alberti G, Zimmet P, Shaw J, et al. Type 2 diabetes in the young: the evolving epidemic: the international diabetes federation consensus workshop. Diabetes Care 2004;27:1798-811.

34. Yan S, Li J, Li S, et al. The expanding burden of cardiometabolic risk in China: the China Health and Nutrition Survey. Obes Rev 2012:13:810-21.

35. Xu Y, Wang L, He J, et al. Prevalence and control of diabetes in Chinese adults. JAMA 2013;310:948-58

36. Corriere M, Rooparinesingh N, Kalyani RR. Epidemiology of diabetes and diabetes complications in the elderly: an emerging public health burden. Curr Diab Rep 2013:13:805-13.

37. Kuswardhani RA, Suastika K. Age and homocystein were risk factor for peripheral arterial disease in elderly with type 2 diabetes mellitus. Acta Med Indones 2010;42:94-9.

38. Pijpers E, Ferreira I, de Jongh RT, et al. Older individuals with diabetes have an increased risk of recurrent falls: analysis of potential mediating factors: the Longitudinal Ageing Study Amsterdam. Age Ageing 2012;41:358-65. 